\title{
Massive and exclusive pontocerebellar damage in mitochondrial disease and NUBPL mutations
}

Figure Brain MRI (1.5 T) and ${ }^{18}$ FDG-PET
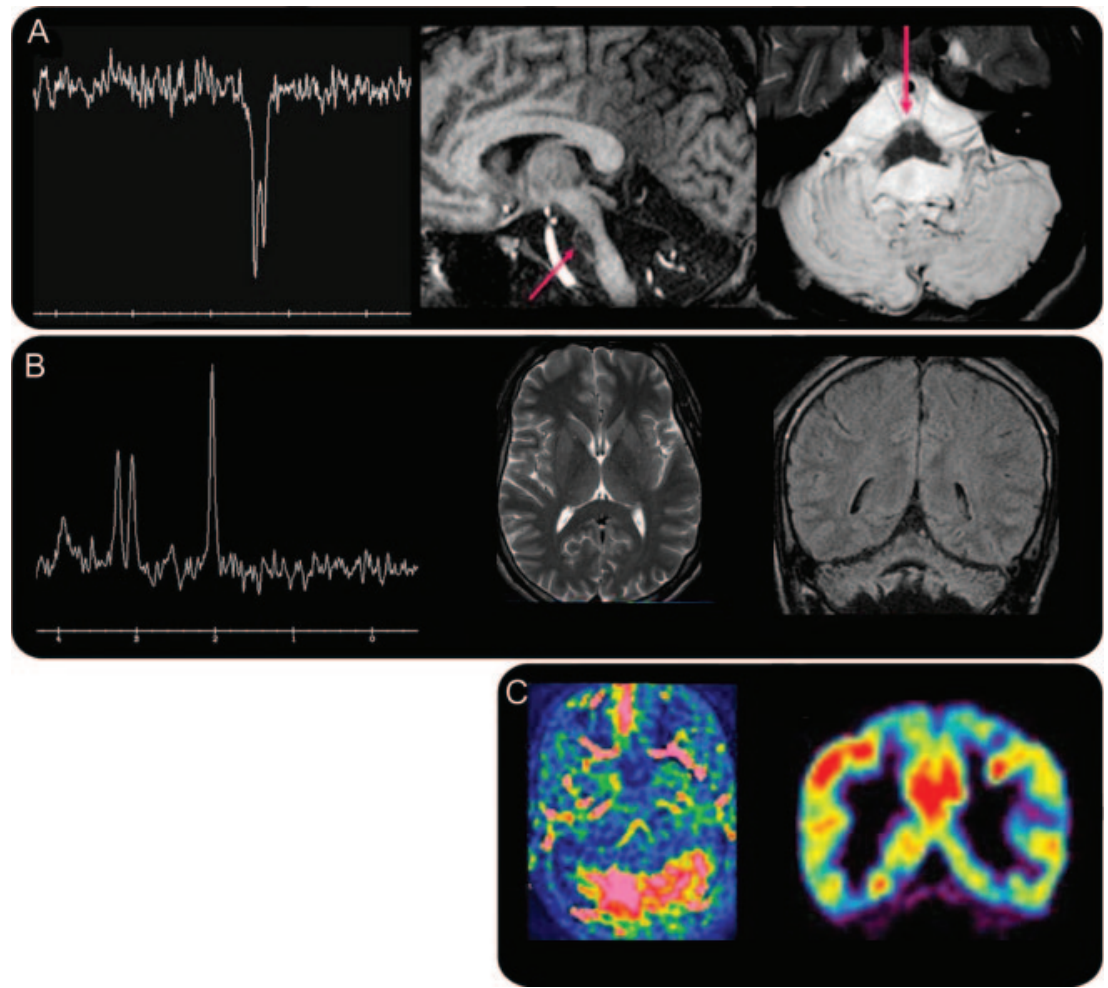

(A) Infratentorial level. MRS-H1-TE144, sagittal T1, axial T2 show lactate peaks with cerebellum and anterior pons anomalies (arrows). (B) Supratentorial level. MRS-H1-TE144, axial T2, coronal fluid-attenuated inversion recovery (FLAIR) show normal MRI. Note cerebellar atrophy on FLAIR. (C) Functional images (cerebellar axial arterial spin labeling, coronal ${ }^{18}$ FDGPET) show increased blood flow and decreased glucose metabolism in the cerebellum and normal supratentorial images.

A 23-year-old man had progressive nystagmus, cerebellar ataxia, pyramidal signs, and slurred speech since toddlerhood. MRI showed T2 hyperintensity of the cerebellum, the anterior brainstem, and the pyramidal tract, sparing the pontine tegmentum (figure, A). Lack of cerebellar NAA and choline on proton magnetic resonance spectroscopy, glucose hypometabolism on ${ }^{18} \mathrm{FDG}-\mathrm{PET}$, and elevated cerebellar lactate suggested mitochondrial disease. ${ }^{1,2}$ Increased cerebellar blood flow suggested vessel proliferation, consistent with mitochondriopathy (figure, C). Skeletal muscle mitochondrial complex I activity was $31 \%$ of control. There was compound heterozygosity for 2 NUBPL mutations: c.205_206delGT, producing a premature stop codon (p.Val69Tyrfs*8); and c.815-217T >C, creating a splicing site and frameshift (p.Asp273Glnfs*32).

Estelle Valerie Tenisch, MD, Anne-Sophie Lebre, MD, PhD, David Grévent, MD, Pascale de Lonlay, MD, PhD, Marlene Rio, MD, PhD, Monica Zilbovicius, MD, PhD, Benoit Funalot, MD, PhD, Isabelle Desguerre, $M D, P h D$, Francis Brunelle, MD, Agnès Rötig, $M D, P h D$, Arnold Munnich, $M D, P h D$, Nathalie Boddaert, $M D, P h D$, Paris, France The authors report no disclosures relevant to the manuscript. Go to Neurology.org for full disclosures.

Correspondence \& reprint requests to Dr. Tenisch: estelle.tenisch@gmail.com

1. Lebre AS, Rio M, Faivre d'Arcier L, et al. A common pattern of brain MRI imaging in mitochondrial diseases with complex I deficiency. J Med Genet 2011;48:16-23.

2. Boddaert N, Romano S, Funalot B, et al. ${ }^{1} \mathrm{H}$ MRS spectroscopy evidence of cerebellar high lactate in mitochondrial respiratory chain deficiency. Mol Genet Metab 2008;93:85-88. 


\section{Neurology}

\section{Massive and exclusive pontocerebellar damage in mitochondrial disease and NUBPL mutations}

Estelle Valerie Tenisch, Anne-Sophie Lebre, David Grévent, et al. Neurology 2012;79;391

DOI 10.1212/WNL.0b013e3182611232

\section{This information is current as of July 23, 2012}

\section{Updated Information \& Services}

References

Citations

Subspecialty Collections

Permissions \& Licensing

\section{Reprints}

including high resolution figures, can be found at: http://n.neurology.org/content/79/4/391.full

This article cites 2 articles, 1 of which you can access for free at: http://n.neurology.org/content/79/4/391.full\#ref-list-1

This article has been cited by 1 HighWire-hosted articles: http://n.neurology.org/content/79/4/391.full\#\#otherarticles

This article, along with others on similar topics, appears in the following collection(s):

Gene expression studies

http://n.neurology.org/cgi/collection/gene_expression_studies Mitochondrial disorders http://n.neurology.org/cgi/collection/mitochondrial_disorders Mitochondrial disorders; see Genetics/Mitochondrial disorders http://n.neurology.org/cgi/collection/mitochondrial_disorders_see_gene tics-mitochondrial_disorders

Information about reproducing this article in parts (figures,tables) or in its entirety can be found online at:

http://www.neurology.org/about/about_the_journal\#permissions

Information about ordering reprints can be found online:

http://n.neurology.org/subscribers/advertise

Neurology ${ }^{\circledR}$ is the official journal of the American Academy of Neurology. Published continuously since 1951, it is now a weekly with 48 issues per year. Copyright Copyright (? 2012 by AAN Enterprises, Inc.. All rights reserved. Print ISSN: 0028-3878. Online ISSN: 1526-632X.

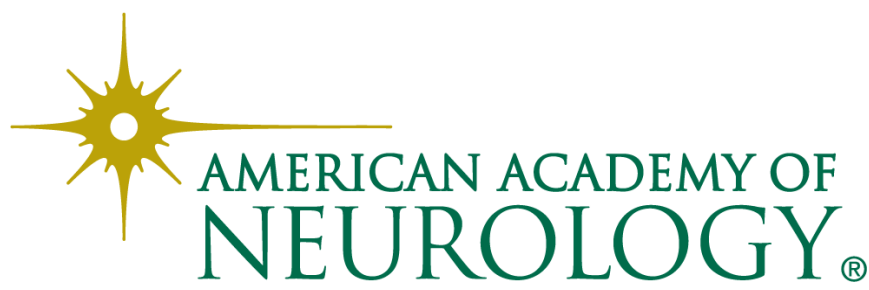

